Original Paper http://ajol.info/index.php/ijbcs http://indexmedicus.afro.who.int

\title{
Apport des descripteurs qualitatifs dans l'identification des accessions de cocotier Grand pour les programmes de régénération des collections au champ
}

\author{
Saraka Didier Martial YAO ${ }^{1}$, Eric-Blanchard Zadjéhi KOFFI ${ }^{*}$, \\ Wentia Alimata Marie-Pierre DARAMCOUM ${ }^{2,3}$, Koffi YOBOUE ${ }^{3,4}$, Jean-Louis Konan \\ KONAN $^{3}$, Nafan DIARRASSOUBA ${ }^{1}$, Roland BOURDEIX ${ }^{5}$ et Raoul Sylvère SIE ${ }^{4}$ \\ ${ }^{1}$ Université Peleforo Gon Coulibaly, UFR Sciences Biologiques, Département de Biochimie-Génétique, Unité \\ Pédagogique et de Recherche (UPR) de Génétique, BP 1328 Korhogo, Côte d'Ivoire. \\ ${ }^{2}$ Université Félix Houphouët Boigny, UFR Biosciences, Laboratoire de Génétique, 22 BP 582 Abidjan 22, Côte d'Ivoire. \\ ${ }^{3}$ CNRA, Station Marc Delorme de Port-Bouët, 07 BP 13 Abidjan 07, Côte d'Ivoire. \\ ${ }^{4}$ Université Nangui-Abrogoua, 02 BP 801 Abidjan 02, Côte d'Ivoire. \\ ${ }^{5}$ CIRAD, Avenue Agropolis 34398, Montpellier Cedex 5, France. \\ *Auteur correspondant ; E-mail: zadjehi2003@yahoo.fr, koffizadjehi@gmail.com
}

\section{RESUME}

Les descripteurs qualitatifs ont été toujours négligés dans les études de caractérisation du cocotier surtout chez les écotypes de type Grand. Cet article se propose de mettre en évidence la contribution de six caractères morphologiques de type qualitatif dans la description et la typologie de 18 populations de cocotier Grand plantées dans la collection internationale de Côte d'Ivoire. Pour y arriver les stipes, les inflorescences, les fleurs femelles et les fruits ont été observés. Compte tenu à la fois de leurs fortes valeurs d'indice de diversité de Shannon normalisé ( $H$ ' variant entre 0,93 et 0,99 ) et faibles valeurs d'indice de diversité de Simpson ( $D$ variant entre 0,24 et 0,28$)$, les caractères formes du fruit et de la noix débourrée ont été les plus discriminants. Les résultats de la classification des populations de cocotier Grand de la collection de Côte d'Ivoire utilisant les caractères qualitatifs sont en désaccord avec la structuration admise et fournie antérieurement à partir des marqueurs morphologiques quantitatifs et moléculaires microsatellites (SSRs). Bien que longtemps marginalisé dans l'étude de la diversité génétique des écotypes Grand de cocotier, l'apport des caractères qualitatifs dans l'identification de ces accessions de la collection de Côte d'Ivoire est discuté. Aussi, l'intérêt du recours aux descripteurs qualitatifs comme alternative aux marqueurs moléculaires pour le suivi de la pureté des accessions de cocotier au champ pendant les cycles de régénération est souligné.

(C) 2020 International Formulae Group. All rights reserved.

Mots clés : Cocos nucifera L., Cocotier Grand, collection, caractérisation, descripteurs qualitatifs, Côte d'Ivoire.

\section{Contribution of qualitative morphological descriptors in the identification of Tall coconut accessions for the programs of field collection regeneration}

\begin{abstract}
Qualitative morphological descriptors have always been neglected in coconut population's characterization, especially in Tall coconut varieties. This paper proposes the contribution of six qualitative traits
\end{abstract}


in the description and typology of 18 Tall coconut populations planted at the international field genebank of Côte d'Ivoire. Relatively to high values of standardized index of Shannon Weaver diversity $H^{\prime}(0.93$ to 0.99$)$ and low values of Simpson diversity index $D(0.24$ to 0.28$)$, the two qualitative traits that are fruit and husked nut shapes were more discriminating. The results about clustering of the Tall coconut populations in Côte d'Ivoire genebank using all studied qualitative characters were in disagreement with the clustering assumed and provided before from quantitative traits and SSRs molecular markers. Although a long time marginalized in the genetic diversity studies, the contribution of the qualitative traits in the identification of Tall coconut populations in international field genebank of Côte d'Ivoire has been discussed. Also, the interest of recourse of the qualitative descriptors as alternative to the molecular markers for purity follow-up of the coconut accession during the cycles of field genebank regeneration is clearly underlined.

(C) 2020 International Formulae Group. All rights reserved.

Keywords: Cocos nucifera L., Tall coconut palms, germplasm, charaterization, qualitative traits, Côte d'Ivoire

\section{INTRODUCTION}

Le cocotier (Cocos nucifera L.) est une monocotylédone pérenne cultivé dans la zone intertropicale humide en raison de son importance socio-économique et la diversité de son utilisation. Il est en effet utilisé comme combustible, matériaux de construction, aliment de bétail, fumure organique, source d'acide gras dans l'industrie oléochimique, etc. Divers pays exploitent la forte diversité existante chez l'espèce Cocos nucifera $\mathrm{L}$. dans leur programme d'amélioration génétique. La collection mondiale compte plus de 300 écotypes de cocotier Grand et Nain décrits et répertoriés selon des marqueurs morphologiques, biochimiques et moléculaires. Dans la collection au champ de Côte d'Ivoire, les marqueurs moléculaires de types biochimique (Benoit et Ghesquière, 1984 ; Jay et al., 1989), RFLP (Lebrun et al., 1998), AFLP (Teulat et al., 2000) et SSR (Konan et al., 2007a; Konan et al., 2011 ; Yao et al., 2013) ainsi que les marqueurs agromorphologiques (Koffi et al., 2013; Yao et al., 2015 ; Koffi et al., 2016; Koffi et al., 2019 ; Daramcoum et al., 2017 ; Yao et al., 2019a ; Yao et al., 2019b) ont largement été utilisés pour sonder la diversité existante au sein et entre les populations de cocotiers Grand, Nain et hybrides. Cependant, les travaux mentionnant l'utilisation des caractères morphologiques qualitatifs pour la caractérisation et la typologie des populations de cocotier Grand sont sommaires et moins nombreux. Par contre, chez les cocotiers Nain, les couleurs du germe, du pétiole, du fruit immature et de l'inflorescence sont utilisées pour définir les écotypes jaune, vert, brun et rouge (De Nuce et Rognon, 1977 ; Le Saint et al., 1983; N'cho et al.,1988). Le gène responsable de la couleur du germe chez le cocotier Nain est gouverné par deux couples d'allèles indépendants $(r, R)$ et $(v, V)$ avec dominance intermédiaire (Bourdeix, 1988). D'après De Nuce et Wuidart (1981) et Sangaré et al. (1984) la couleur du fruit immature n'est pas une caractéristique importante des cocotiers Grand. Cependant, De Nuce et Wuidart (1979) ont mentionné que bien que la couleur du fruit immature des écotypes Grand soit moins tranchée, elle peut permettre de déceler des plants illégitimes. Aussi, ces travaux ont révélé que les formes du fruit et de la noix débourrée permettent d'identifier certaines populations de cocotier Grand. Vu que l'apport des caractères morphologiques de type qualitatif dans l'identification des populations de cocotier Grand n'est pas négligeable, l'exploration de nouveaux moyens de sonder la diversité du cocotier permettrait de connaitre davantage les différentes populations de cocotier Grand disponibles en collection. Les progrès désirés ont d'autant plus de chance d'être atteints que si le sélectionneur et/ou le gestionnaire d'une collection possède une vision claire et précise des caractéristiques propres de chaque population de cocotier Grand. De plus, la 
méconnaissance de certains critères d'identification morphologique d'une population de cocotier est préjudiciable pour sa régénération sans risque d'altérer sa pureté. L'objectif de cette étude est d'utiliser les caractères morphologiques de type qualitatif pour décrire simultanément plusieurs populations de cocotier Grand et apprécier pour la première fois leur typologie.

\section{MATERIEL ET METHODES \\ Matériel végétal}

L'étude a porté sur 18 accessions de cocotier Grand issues d'un premier cycle de régénération (G1) par la méthode de fécondation contrôlée (De Nuce et al., 1980 ; Konan et al., 2008). Ces accessions sont conservées dans la collection internationale pour l'Afrique et l'Océan Indien sise à la station Marc Delorme de Port-Bouët à Abidjan, Côte d'Ivoire (Figure1). Ces accessions régénérées de cocotier Grand ont une faible différenciation génétique avec celles initialement introduites (G0) (Yao et al., 2013). Elles ont été plantées à la densité de 143 individus.ha ${ }^{-1}$ sur les parcelles numérotées 043 , 091 et 081 (Tableau 1) respectivement en 1995, 1998 et 2002.

\section{Mesure des caractères morphologiques qualitatifs}

Suivant les effectifs d'arbres vivants au champ, des effectifs de 20 à 30 individus sains ont été aléatoirement sélectionnés par accession. Une observation visuelle du bulbe $\left(B b \_x\right)$ à la base du stipe a permis de le classifier selon IPGRI (1995) en 3 phénotypes que sont absent, moyen et élevé (Tableau 2). Sur l'inflorescence de rang 11, reconnaissable par le bout nécrosé des fleurs femelles qu'elle porte, la coloration du pédoncule floral (CPF_x) et des fleurs femelles (CFF_x) ont été notées suivant les 4 modalités que sont jaune, rouge, verte et brune (IPGRI, 1995). Suivant ces mêmes modalités la couleur du fruit immature (Fim_x) a été codifiée sur l'inflorescence de rang 20 (IPGRI, 1995;
Bioversity International, 2008 ; Yao et al., 2015). Un fruit mature âgé de 12 mois porté par le régime de rang 24 de chaque individu a été aléatoirement choisi. La forme du fruit $\left(\mathrm{F}_{-} \mathrm{x}\right) \mathrm{a}$ été codifiée suivant les 4 modalités que sont anguleuse, oblongue, ovoïde et ronde (IPGRI, 1995; Yao et al., 2015). Ce fruit mature a été débourré à l'aide d'un pieu métallique et la forme de la noix (N_x) qu'elle contient a été classifiée suivant les 4 phénotypes aplatie aux pôles, ovoïde, pointée et ronde (Bioversity International, 2008).

\section{Analyses statistiques}

D'abord, la variabilité des 6 caractères qualitatifs étudiés a été évaluée en déterminant la fréquence d'apparition $(P i)$ de chacune des modalités. Egalement, pour chaque caractère qualitatif les indices de diversité de ShannonWeaver normalisé $\left(H^{\prime}\right)$ et de Simpson $(D)$ variant de 0 à 1 ont été calculés (Baudouin et Santos, 2005 ; Arunachalam et al., 2005, Yao et al., 2015) :

$P i=\frac{N i}{N}$

$H^{\prime}=\frac{\sum_{i}^{n}(P i) \mathrm{x} \ln (P i)}{\ln (n)}$

$D=\frac{\sum_{i}^{n} N i(N i-1)}{N(N-1)}$

Avec $\mathrm{Ni}$ l'effectif observé de l'ì̀me modalité pour un caractère donné, $N$ l'effectif total des individus et $n$ le nombre de modalité d'un caractère. Lorsque $H^{\prime}$ tend vers 1 , la diversité d'un caractère est élevée au sein ou entre les populations de cocotier Grand étudiées (Yao et al., 2015). Lorsque $D$ tend vers 0 , la probabilité que deux individus tirés au hasard dans la collection ou dans une population de cocotier Grand aient le même phénotype pour un caractère donné est faible, en d'autres termes la variabilité du caractère est élevée (Yao et al., 2015). Ensuite, à chaque population de cocotier Grand a été associée sa fréquence d'appartenance à une modalité et une Analyse Factorielle de Correspondance (AFC) a été réalisée. L'AFC réalisée sur le logiciel Statistica version 7.1 (StatSoft, 2007) 
pour chacun des six caractères qualitatifs étudiés a permis de structurer les populations de cocotier Grand suivant les phénotypes les plus fréquemment rencontrés. Enfin, une matrice de donnée binaire codée suivant la présence (1) et l'absence (0) des modalités de chaque caractère par population a été construite. A partir donc de l'ensemble des six caractères qualitatifs étudiés, l'indice de similarité de Jaccard a été calculé pour chaque paire de population et un dendrogramme structurant les populations de cocotier Grand suivant les phénotypes communs qu'elles expriment a été construit sur le logiciel XLSTAT version 2015.

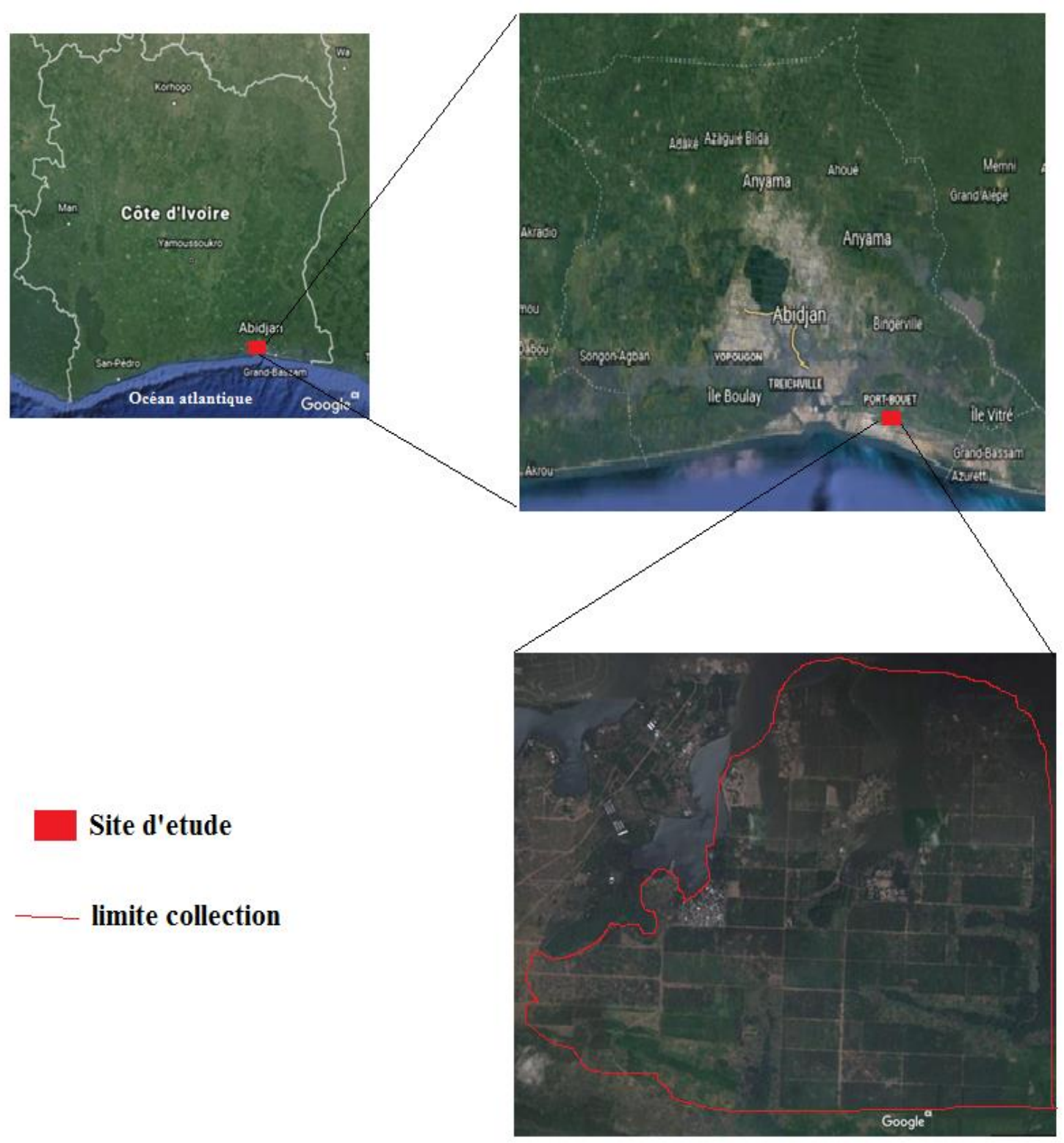

Figure 1. Localisation des parcelles de conservation des accessions de cocotier dans la collection internationale pour l'Afrique et l'Océan Indien sise à la station Marc Delorme de Port-Bouët à Abidjan, Côte d'Ivoire. 
Tableau 1 : Désignation, code et localisation des 18 accessions régénérées (G1) de cocotier Grand dans la collection internationale pour l'Afrique et l'Océan Indien.

\begin{tabular}{|c|c|c|c|c|c|}
\hline $\mathbf{N}^{\circ}$ & Population de cocotier Grand & Codes & $\mathbf{n}^{\circ}$ parcelle $^{\mathrm{a}}$ & \multicolumn{2}{|c|}{ Pays, Groupe géographique } \\
\hline 1 & Grand Ouest Africain & GOA G1 & 091 & \multicolumn{2}{|c|}{ Côte d'Ivoire, Afrique } \\
\hline 2 & Grand Sri Lanka & GSL G1 & 081 & \multicolumn{2}{|c|}{ Sri Lanka, Océan Indien } \\
\hline 3 & Grand Rotuma & GRT G1 & 081 & \multicolumn{2}{|c|}{ Iles Fidji, Pacifique Sud } \\
\hline 4 & Grand Tonga & GTG G1 & 081 & \multicolumn{2}{|c|}{ Iles Tonga, Pacifique Sud } \\
\hline 5 & Grand Vanuatu & GVT G1 & 081 & \multicolumn{2}{|c|}{ Vanuatu, Pacifique Sud } \\
\hline 6 & Grand Tagnaman & GTN G1 & 081 & \multicolumn{2}{|c|}{ Philippines, Extrême-Orient } \\
\hline & \multicolumn{5}{|l|}{ Population Grand Thaïlande } \\
\hline 7 & Grand Thaïlande Sawi & GTH1 G1 & 091 & \multicolumn{2}{|c|}{ Thaïlande, Extrême-Orient } \\
\hline \multirow[t]{2}{*}{8} & Grand Thaïlande Ko Samui & \multirow[t]{2}{*}{ GTH4 G1 } & \multirow[t]{2}{*}{091} & \multicolumn{2}{|c|}{ Thaïlande, Extrême-Orient } \\
\hline & Population Grand Polynésie & & & \\
\hline 9 & Grand Polynésie Tahiti & GPY1 G1 & 091 & Polyı & lésie, Pacifique Sud \\
\hline \multirow[t]{2}{*}{10} & Grand Polynésie Rangiora & GPY2 G1 & 091 & \multicolumn{2}{|c|}{ Polynésie, Pacifique Sud } \\
\hline & \multicolumn{5}{|l|}{ Population Grand Cambodge } \\
\hline 11 & Grand Cambodge Kopal' Tani & GCB4 G1 & 043 & \multicolumn{2}{|c|}{ Cambodge, Extrême-Orient } \\
\hline 12 & $\begin{array}{l}\text { Grand Cambodge Feu' Kompong } \\
\text { Trach }\end{array}$ & GCB5 G1 & 043 & \multicolumn{2}{|c|}{ Cambodge, Extrême-Orient } \\
\hline 13 & Grand Cambodge Ream & GCB7 G1 & 091 & \multicolumn{2}{|c|}{ Cambodge, Extrême-Orient } \\
\hline 14 & Grand Cambodge Sre Chan & GCB8 G1 & 091 & \multicolumn{2}{|c|}{ Cambodge, Extrême-Orient } \\
\hline 15 & Grand Cambodge Battambang & GCB9 G1 & 091 & \multicolumn{2}{|c|}{ Cambodge, Extrême-Orient } \\
\hline 16 & Grand Cambodge Koh Rong & $\begin{array}{l}\text { GCB10 } \\
\text { G1 }\end{array}$ & 091 & \multicolumn{2}{|c|}{ Cambodge, Extrême-Orient } \\
\hline & \multicolumn{5}{|l|}{ Population Grand Salomon } \\
\hline 17 & Grand Salomon & GSN G1 & 091 & \multicolumn{2}{|c|}{ Iles Salomons, Pacifique Sud } \\
\hline 18 & Grand Rennel & GRL G1 & 091 & \multicolumn{2}{|c|}{ Iles Salomons, Pacifique Sud } \\
\hline \multicolumn{6}{|c|}{$\begin{array}{l}{ }^{a} \text { Parcelle } n^{\circ} 043 \text { (Latitude Nord } 05^{\circ} 15.781^{\prime} \text { - Longitude Ouest } 003^{\circ} 50.220^{\prime} \text { ') } \\
\text { Parcelle } n^{\circ} 081 \text { (Latitude Nord } 05^{\circ} 16.361^{\prime} \text { - Longitude Ouest } 003^{\circ}{ }^{\circ} 0.587^{\prime} \text { ) } \\
\text { Parcelle } n^{\circ} 091 \text { (Latitude Nord } 05^{\circ} 16.494^{\prime} \text { - Longitude Ouest } 003^{\circ} 50.754^{\prime} \text { ) }\end{array}$} \\
\hline \multicolumn{6}{|c|}{$\begin{array}{l}\text { Tableau 2. Liste des six descripteurs qualitatifs évalués, leur symbole et code des phénotypes } \\
\text { observés sur } 18 \text { accessions régénérées (G1) de cocotier Grand dans la collection internationale pour } \\
\text { l'Afrique et l'Océan Indien. }\end{array}$} \\
\hline \multicolumn{2}{|r|}{ Descripteurs mesurés } & Symbole & \multicolumn{2}{|c|}{ Phénotypes observés } & Code du phénotype \\
\hline \multirow[t]{3}{*}{ Stip } & Catégorie du bulbe & $\mathrm{Bb} \_\mathrm{x}$ & \multicolumn{2}{|l|}{ Absence } & 0 \\
\hline & & & Moyen & & 3 \\
\hline & & & Elevé & & 7 \\
\hline
\end{tabular}




\begin{tabular}{|c|c|c|c|c|}
\hline \multirow[t]{8}{*}{ Inflorescence } & Couleur Fleur Femelle & $\overline{\text { CFF_x }}$ & Jaune & 1 \\
\hline & & & Rouge & 4 \\
\hline & & & Verte & 7 \\
\hline & & & Brune & 10 \\
\hline & Couleur Pédoncule Floral & $\overline{\text { CPF_x }}$ & Jaune & 1 \\
\hline & & & Rouge & 4 \\
\hline & & & Verte & 7 \\
\hline & & & Brune & 10 \\
\hline \multirow[t]{12}{*}{ Fruit } & Forme Fruit & $\bar{F} F_{-} x$ & Oblongue & 1 \\
\hline & & & Ovoïde & 2 \\
\hline & & & Anguleuse & 3 \\
\hline & & & Ronde & 4 \\
\hline & Forme Noix Débourrée & N_x & Pointée & 2 \\
\hline & & & Ovoïde & 4 \\
\hline & & & Ronde & 6 \\
\hline & & & Aplatie aux pôles & 8 \\
\hline & Couleur Fruit Immature & CFim_x & Jaune & 1 \\
\hline & & & Rouge & 4 \\
\hline & & & Verte & 7 \\
\hline & & & Brune & 10 \\
\hline
\end{tabular}

x modalité d'un descripteur qualitatif.

\section{RESULTATS}

\section{Variabilité de la catégorie du bulbe}

Chez GOA G1, GPY1 G1, GCB4 G1, GCB5 G1 et GRL G1, les trois phénotypes que sont absent ( 8,7 à $31,8 \%)$, moyen (15 à 66,7\%) et élevé $(23,3$ à $65,2 \%)$ ont été présents. L'indice de diversité de Shannon Weaver normalisé $\left(H^{\prime}\right)$ a varié de 0,76 (GOA G1 et GPY1 G1) à 0,98 (GCB4 G1) avec une moyenne de 0,6. La probabilité que deux individus tirés au hasard dans une population de cocotier Grand présentent la même catégorie de bulbe $(D)$ a varié entre 0,25 (GCB4 G1) et 1 (GTH4 G1, GCB9 G1 et GCB10 G1). Dans l'ensemble de la collection, la valeur moyenne de $D$ a été de 0,61 (Tableau 3 ; Tableau 4). L'AFC a révélé qu'une forte proportion d'individus GOA G1 $(66,7 \%)$ présente le phénotype bulbe moyen. Excepté chez GTH4 G1, GCB9 G1 et GCB10 G1, bien que le phénotype bulbe moyen soit présent, le phénotype bulbe élevé a été le plus rencontré à des fréquences variantes entre 45,5 et $100 \%$ (Figure 2).

\section{Variabilité des couleurs du fruit immature, des fleurs femelles et du pédoncule floral}

Toutes les modalités de la couleur des fruits ont été observées chez les populations GRT G1, GVT G1, GTH4 G1, GPY1 G1 et GCB9 G1. En effet, une proportion de 4,3 à $16,7 \%$ de ces individus a donné des fruits immatures rouges, 11,1 à $36,7 \%$ des fruits bruns, 46,7 à $72,2 \%$ des fruits verts et 5,3 à $10 \%$ des fruits jaunes. $H^{\prime}$ a varié de 0,53 (GTH4 G1) à 0,8 (GCB9 G1) avec une moyenne de 0,65 . $D$ a varié entre 0,35 (GVT G1) et 0,9 (GCB7 G1) avec une moyenne de 0,5 (Tableaux 3 et 4). L'AFC a révélé que seulement GTN G1 et GSN G1 ont présenté une proportion élevée d'individus avec des fruits immatures respectivement de couleurs brune $(53,3 \%)$ et rouge $(50 \%)$. Les colorations verte et brune du fruit immature ont été observées chez les autres populations de cocotier Grand (Figure 3a).

Chez les populations GVT G1, GTN G1, GPY1 G1, GSN G1 et GRL G1 les individus ont exprimés les 4 colorations (rouge 
$(3,3$ à $10 \%)$, brune $(8,7$ à $50 \%)$, verte $(13,6$ à $73,9 \%)$ et jaune (13 à 40\%)) des fleurs femelles. Pendant que $H^{\prime}$ a varié entre 0,6 (GPY1 G1) et 0,92 (GSN G1), $D$ a varié entre 0,26 (GSN G1) et 0,55 (GPY1 G1). Les valeurs de $H$ ' et $D$ ont été respectivement de 0,8 et 0,36 dans l'ensemble de la collection (Tableau 3 et 4). L'AFC a révélé que les colorations jaune, verte et brune des fleurs femelles ont coexisté avec une légère prédominance de la coloration jaune chez GTH1 G1, GCB10 G1 et GPY2 G1, de la coloration brune chez GOA G1 et GRL G1 et de la coloration verte chez les autres populations (Figure $3 b$ ).

Des proportions de 3,3\%, 26,7\% et 3,3\% d'individus GVT G1 ont respectivement les 4 phénotypes rouge, brune, verte et jaune de la coloration du pédoncule floral. Chez GVT G1, $H^{\prime}$ et $D$ ont été respectivement de 0,61 et 0,5 avec successivement des valeurs moyennes de 0,47 et 0,64 . Les individus GCB7 G1 et GCB10 G1 ont tous des pédoncules floraux de couleur verte (Tableau 3 ; Tableau 4). L'AFC a révélé que chez les populations étudiées les couleurs rouge, verte et brune du pédoncule floral ont été fréquentes avec une légère prédominance du vert excepté chez GSN G1 où le brun a dominé (Figure 3c).

\section{Variabilité de la forme du fruit mature}

En majorité, chez les populations étudiées, les formes anguleuse, oblongue, ovoïde et ronde du fruit mature coexistent. L'absence de la forme anguleuse du fruit a été notée chez GTH4 G1, GCB4 G1 et GCB5 G1. Les individus GRT G1 n'ont pas présenté des fruits de forme ronde (Tableau 3). Au sein des populations de cocotier Grand étudiées $H^{\prime}$ a varié de 0,7 (GRL G1) à 0,91 (GSL G1) avec une moyenne de $0,99 . D$ a varié entre 0,28 (GPY1 G1) et 0,46 (GRT G1). Dans l'ensemble de la collection la valeur de $D$ a été de 0,24 (Tableau 4). L'AFC a révélé que la forme oblongue du fruit a été plus fréquente chez GRT G1, GTG G1 et GRL G1. Une proportion élevée d'individus GOA G1, GSL G1 et GSN G1 ont donné des fruits anguleux. Les fruits de forme ronde ont été fréquemment rencontrés chez GTN G1, GTH1 G1, GTH4 G1, GCB4 G1, GCB5 G1, GCB8 G1 et GCB9
G1. La forme ovoïde du fruit mature a été plus répandue chez GCB7 G1 et GCB10 (Figure 4).

Variabilité de la forme de la noix débourrée Les individus GOA G1, GSL G1, GRT G1, GTG G1, GPY1 G1, GPY2 G1, GCB8 G1, GCB9 G1, GCB10 G1, GSN G1 et GRL G1 ont présenté toutes les modalités de la forme des noix débourrées. Ils ont produit des fruits contenant des noix de forme aplatie aux pôles (16,7 à $45,5 \%)$, ovoïde ( 16,7 à $45,5 \%)$, pointée (3,3 à 26,7\%) et ronde $(6,7$ à 44,4\%). Au sein de ces populations $H^{\prime}$ a varié de 0,74 (GCB10 G1) à 0,98 (GOA G1) avec une moyenne de 0,93 tandis que $\mathrm{D}$ a varié entre 0,23 (GOA G1) et 0,63 (GTH1 G1) avec une moyenne de 0,28 dans la collection (Tableau 4). L'absence de la forme pointée de la noix débourrée a été observée chez GVT G1, GTN G1, GTH1 G1, GTH4 G1, GCB5 G1 et GCB7 G1. La forme ovoïde de la noix débourrée n'a pas été observée chez GCB4 G1 et GCB5 G1 (Tableau 3 et 4). L'AFC a révélé que la forme aplatie aux pôles de la noix débourrée a été plus fréquente chez GTN G1, GSL G1, GVT G1, GPY1 G1, GSN G1 et GCB9 G1. Seulement, GOA G1 a produit plus de fruits contenant des noix de forme pointée. La forme ovoïde des noix débourrées a été plus répandue chez GPY2 G1, GCB7 G1 et GRL G1 (Figure 5).

\section{Structure de la diversité des populations de cocotier Grand}

Le dendrogramme réalisé à partir des 6 caractères qualitatifs pris ensemble a partitionné les 18 populations de cocotier Grand étudiées en 5 classes phénotypiques à $88 \%$ de similarité (Figure 6). Suivant les 6 caractères qualitatifs étudiés, les populations de cocotier Grand originaires de l'ExtrêmeOrient et celles regroupées dans la classe phénotypique 1 ont été les plus diversifiées génétiquement (Figure 6). Le dendrogramme a aussi montré que certaines populations de cocotier Grand collectées dans le même pays comme les populations Grand de Cambodge (GCB) et Grand de Thaïlande (GTH) n'ont pas été classifiées dans le même groupe (Figure 6). 
Tableau 3 : Fréquences (\%) d'apparition des différentes modalités des caractères qualitatifs chez 18 accessions régénérées (G1) de cocotier Grand dans la Collection Internationale pour l'Afrique et l'Océan Indien.

\begin{tabular}{|c|c|c|c|c|c|c|c|c|c|c|c|c|c|c|c|c|c|c|c|}
\hline \multicolumn{2}{|c|}{ Caractères qualitatifs } & $\begin{array}{c}\text { GO } \\
\text { A } \\
\text { G1 }\end{array}$ & $\begin{array}{c}\text { GSL } \\
\text { G1 }\end{array}$ & $\begin{array}{c}\text { GRT } \\
\text { G1 }\end{array}$ & $\begin{array}{c}\text { GT } \\
\text { G } \\
\text { G1 }\end{array}$ & $\begin{array}{c}\text { GV } \\
\text { T } \\
\text { G1 }\end{array}$ & $\begin{array}{c}\text { GTN } \\
\text { G1 }\end{array}$ & $\begin{array}{c}\text { GTH } \\
\mathbf{1} \\
\text { G1 }\end{array}$ & $\begin{array}{c}\text { GTH } \\
4 \\
\text { G1 }\end{array}$ & $\begin{array}{c}\text { GPY1 } \\
\text { G1 }\end{array}$ & $\begin{array}{c}\text { GPY2 } \\
\text { G1 }\end{array}$ & $\begin{array}{c}\text { GCB4 } \\
\text { G1 }\end{array}$ & $\begin{array}{c}\text { GCB5 } \\
\text { G1 }\end{array}$ & $\begin{array}{c}\text { GCB7 } \\
\text { G1 }\end{array}$ & $\begin{array}{c}\text { GCB8 } \\
\text { G1 }\end{array}$ & $\begin{array}{c}\text { GCB9 } \\
\text { G1 }\end{array}$ & $\begin{array}{c}\text { GCB10 } \\
\text { G1 }\end{array}$ & $\begin{array}{c}\text { GSN } \\
\text { G1 }\end{array}$ & $\begin{array}{c}\text { GRL } \\
\text { G1 }\end{array}$ \\
\hline \multirow{3}{*}{ Forme bulbe } & Absent & 10 & 0 & 0 & 0 & 0 & 0 & 0 & 0 & 8,7 & 0 & 25 & 26,3 & 0 & 0 & 0 & 0 & 0 & 31,8 \\
\hline & Moyen & 66,7 & 23,3 & 10 & 13,3 & 3,3 & 6,7 & 10,5 & 0 & 26,1 & 11,8 & 37,5 & 15,8 & 5 & 22,2 & 0 & 0 & 15 & 45,5 \\
\hline & Elevé & 23,3 & 76,7 & 90 & 86,7 & 96,7 & 93,3 & 89,5 & 100 & 65,2 & 88,2 & 37,5 & 57,9 & 95 & 77,8 & 100 & 100 & 85 & 22,7 \\
\hline \multirow{4}{*}{$\begin{array}{l}\text { Couleur fruit } \\
\text { immature }\end{array}$} & Rouge & 3,3 & 0 & 16,7 & 0 & 13,3 & 0 & 5,3 & 5,3 & 4,3 & 17,6 & 0 & 0 & 0 & 0 & 11,1 & 0 & 50 & 18,2 \\
\hline & Brune & 43,3 & 36,7 & 13,3 & 33,3 & 36,7 & 53,3 & 0 & 10,5 & 21,7 & 11,8 & 25 & 21,1 & 5 & 16,7 & 11,1 & 0 & 5 & 22,7 \\
\hline & Verte & 53,4 & 63,3 & 60 & 60 & 46,7 & 46,7 & 89,5 & 78,9 & 65,3 & 70,6 & 75 & 78,9 & 95 & 83,3 & 72,2 & 94,4 & 45 & 59,1 \\
\hline & Jaune & 0 & 0 & 10 & 6,7 & 3,3 & 0 & 5,3 & 5,3 & 8,7 & 0 & 0 & 0 & 0 & 0 & 5,6 & 5,6 & 0 & 0 \\
\hline \multirow{4}{*}{$\begin{array}{l}\text { Couleur fleurs } \\
\text { femelles }\end{array}$} & Rouge & 0 & 0 & 0 & 0 & 3,3 & 6,7 & 5,3 & 0 & 4,4 & 11,7 & 0 & 0 & 0 & 0 & 0 & 0 & 10 & 4,5 \\
\hline & Brune & 36,7 & 13,3 & 20 & 20 & 33,4 & 30 & 0 & 21,1 & 8,7 & 0 & 25 & 21,1 & 5 & 5,6 & 27,8 & 0 & 40 & 50 \\
\hline & Verte & 30 & 53,4 & 56,7 & 53,3 & 23,3 & 46,7 & 57,9 & 63,2 & 73,9 & 41,2 & 50 & 63,2 & 70 & 61,1 & 50 & 44,4 & 20 & 13,6 \\
\hline & Jaune & 33,3 & 33,3 & 23,3 & 26,7 & 40 & 16,7 & 36,8 & 15,7 & 13 & 47,1 & 25 & 15,8 & 25 & 33,3 & 22,2 & 55,6 & 30 & 31,8 \\
\hline
\end{tabular}


S. D. M. YAO et al. /Int. J. Biol. Chem. Sci. 14(2): 580-599, 2020

\begin{tabular}{|c|c|c|c|c|c|c|c|c|c|c|c|c|c|c|c|c|c|c|c|}
\hline & Rouge & 0 & 0 & 0 & 0 & 3,3 & 0 & 0 & 0 & 0 & 5,9 & 0 & 0 & 0 & 0 & 0 & 0 & 5 & 0 \\
\hline \multirow{3}{*}{$\begin{array}{l}\text { Couleur } \\
\text { pédoncule } \\
\text { floral }\end{array}$} & Brune & 20 & 13,3 & 16,7 & 20 & 26,7 & 43,3 & 5,2 & 5,3 & 13 & 11,8 & 25 & 21,1 & 0 & 5,6 & 16,7 & 0 & 55 & 40,9 \\
\hline & Verte & 76,7 & 80 & 80 & 53,3 & 66,7 & 53,4 & 73,7 & 78,9 & 87 & 82,3 & 75 & 78,9 & 100 & 94,4 & 83,3 & 100 & 40 & 59,1 \\
\hline & Jaune & 3,3 & 6,7 & 3,3 & 26,7 & 3,3 & 3,3 & 21,1 & 15,8 & 0 & 0 & 0 & 0 & 0 & 0 & 0 & 0 & 0 & 0 \\
\hline \multirow{6}{*}{ Forme Fruit } & Anguleuse & 46,7 & 46,7 & 33,3 & 10 & 36,7 & 10 & 10,5 & 0 & 21,7 & 29,3 & 0 & 0 & 5 & 11,1 & 11,1 & 5,6 & 40 & 22,7 \\
\hline & Oblongue & 13,3 & 20 & 60 & 53,3 & 43,3 & 13,3 & 5,3 & 21,1 & 43,5 & 47,1 & 12,5 & 36,8 & 25 & 11,1 & 11,1 & 5,6 & 15 & 63,6 \\
\hline & Ovoïde & 33,3 & 20 & 6,7 & 16,7 & 10 & 23,3 & 31,6 & 21,1 & 26,1 & 11,8 & 37,5 & 10,5 & 50 & 16,7 & 33,3 & 50 & 40 & 9,1 \\
\hline & Ronde & 6,7 & 13,3 & 0 & 20 & 10 & 53,3 & 52,6 & 57,8 & 8,7 & 11,8 & 50 & 52,6 & 20 & 61,1 & 44,5 & 38,9 & 5 & 4,5 \\
\hline & $\begin{array}{l}\text { Aplatie aux } \\
\text { pôles }\end{array}$ & 26,7 & 40 & 43,3 & 33,3 & 50 & 40 & 15,8 & 42,1 & 47,8 & 23,5 & 12,5 & 47,4 & 25 & 22,2 & 55,6 & 22,2 & 35 & 36,4 \\
\hline & Ovoïde & 26,7 & 20 & 43,3 & 20 & 6,7 & 23,3 & 5,3 & 10,5 & 21,7 & 47,1 & 0 & 0 & 40 & 33,3 & 16,7 & 27,8 & 25 & 45,5 \\
\hline \multirow{2}{*}{$\begin{array}{l}\text { Forme noix } \\
\text { débourrée }\end{array}$} & Pointée & 30 & 6,7 & 6,7 & 3,3 & 0 & 0 & 0 & 0 & 8,7 & 5,9 & 12,5 & 0 & 0 & 5,6 & 5,6 & 5,6 & 10 & 4,5 \\
\hline & Ronde & 16,6 & 33,3 & 6,7 & 43,3 & 43,3 & 36,7 & 78,9 & 47,4 & 21,8 & 23,5 & 75 & 52,6 & 35 & 38,9 & 22,2 & 44,4 & 30 & 13,6 \\
\hline
\end{tabular}


Tableau 4. Indices de diversité de Shannon Weaver normalisé $\left(H^{\prime}\right)$ et de diversité de Simpson $(D)$ des caractères qualitatifs observés sur 18 accessions régénérées $(\mathrm{G} 1)$ de cocotier Grand dans la Collection Internationale pour l'Afrique et l'Océan Indien.

\section{Caractères qualitatifs}

\begin{tabular}{|c|c|c|c|c|c|c|c|}
\hline $\begin{array}{l}\text { Accessions de } \\
\text { cocotier Grand }\end{array}$ & Indices de diversité & Forme bulbe & $\begin{array}{l}\text { Couleur fruit } \\
\text { immature }\end{array}$ & $\begin{array}{l}\text { Couleur fleurs } \\
\text { femelles }\end{array}$ & $\begin{array}{c}\text { Couleur pédoncule } \\
\text { floral }\end{array}$ & Forme Fruit & $\begin{array}{c}\text { Forme noix } \\
\text { débourrée }\end{array}$ \\
\hline \multirow[t]{2}{*}{ GOA G1 } & $H^{\prime}$ & 0,76 & - & - & - & 0,84 & 0,98 \\
\hline & $D$ & 0,49 & 0,45 & 0,31 & 0,62 & 0,33 & 0,23 \\
\hline \multirow[t]{2}{*}{ GSL G1 } & $H^{\prime}$ & - & - & - & - & 0,91 & 0,78 \\
\hline & $D$ & 0,63 & 0,52 & 0,39 & 0,65 & 0,29 & 0,29 \\
\hline \multirow[t]{2}{*}{ GRT G1 } & $H^{\prime}$ & - & 0,79 & - & - & - & 0,78 \\
\hline & $D$ & 0,81 & 0,40 & 0,40 & 0,66 & 0,46 & 0,36 \\
\hline \multirow[t]{2}{*}{ GTG G1 } & $H^{\prime}$ & - & - & - & - & 0,85 & 0,83 \\
\hline & $D$ & 0,76 & 0,46 & 0,37 & 0,37 & 0,34 & 0,32 \\
\hline \multirow[t]{2}{*}{ GVT G1 } & $H^{\prime}$ & - & 0,79 & 0,85 & 0,61 & 0,85 & - \\
\hline & $D$ & 0,93 & 0,35 & 0,30 & 0,50 & 0,32 & 0,42 \\
\hline \multirow[t]{2}{*}{ GTN G1 } & $H^{\prime}$ & - & - & 0,86 & - & 0,84 & - \\
\hline & $D$ & 0,87 & 0,49 & 0,32 & 0,46 & 0,34 & 0,33 \\
\hline \multirow[t]{2}{*}{ GTH1 G1 } & $H^{\prime}$ & - & - & - & - & 0,78 & - \\
\hline & $D$ & 0,80 & 0,80 & 0,44 & 0,57 & 0,36 & 0,63 \\
\hline \multirow[t]{2}{*}{ GTH4 G1 } & $H^{\prime}$ & - & 0,53 & - & - & - & - \\
\hline & $D$ & 1 & 0,62 & 0,44 & 0,63 & 0,39 & 0,38 \\
\hline GPY1 G1 & $H^{\prime}$ & 0,76 & 0,69 & 0,60 & - & 0,90 & 0,88 \\
\hline
\end{tabular}


S. D. M. YAO et al. /Int. J. Biol. Chem. Sci. 14(2): 580-599, 2020

\begin{tabular}{|c|c|c|c|c|c|c|c|}
\hline & $D$ & 0,48 & 0,46 & 0,55 & 0,76 & 0,28 & 0,30 \\
\hline \multirow[t]{2}{*}{ GPY2 G1 } & $H^{\prime}$ & - & - & - & - & 0,87 & 0,86 \\
\hline & $D$ & 0,78 & 0,51 & 0,37 & 0,68 & 0,29 & 0,29 \\
\hline \multirow[t]{2}{*}{ GCB4 G1 } & $H^{\prime}$ & 0,98 & - & - & - & - & - \\
\hline & $D$ & 0,25 & 0,57 & 0,29 & 0,57 & 0,32 & 0,54 \\
\hline \multirow[t]{2}{*}{ GCB5 G1 } & $\overline{H^{\prime}}$ & 0,87 & - & - & - & - & - \\
\hline & $D$ & 0,40 & 0,65 & 0,44 & 0,65 & 0,39 & 0,47 \\
\hline \multirow[t]{2}{*}{ GCB7 G1 } & $H^{\prime}$ & - & - & - & - & - & 0,84 \\
\hline & $D$ & 0,90 & 0,90 & 0,53 & 1 & 0,32 & 0,31 \\
\hline \multirow[t]{2}{*}{ GCB8 G1 } & $H^{\prime}$ & - & - & - & - & - & 0,78 \\
\hline & $D$ & 0,63 & 0,71 & 0,46 & 0,89 & 0,39 & 0,27 \\
\hline \multirow[t]{2}{*}{ GCB9 G1 } & $H^{\prime}$ & - & 0,80 & - & - & - & 0,87 \\
\hline & $D$ & 1 & 0,52 & 0,34 & 0,71 & 0,29 & 0,35 \\
\hline \multirow[t]{2}{*}{ GCB10 G1 } & $H^{\prime}$ & - & - & - & - & - & 0,74 \\
\hline & $D$ & 1 & 0,89 & 0,48 & 1 & 0,37 & 0,29 \\
\hline \multirow[t]{2}{*}{ GSN G1 } & $H^{\prime}$ & - & - & 0,92 & - & 0,84 & 0,94 \\
\hline & $D$ & 0,73 & 0,43 & 0,26 & 0,44 & 0,31 & 0,25 \\
\hline \multirow[t]{2}{*}{ GRL G1 } & $H^{\prime}$ & 0,96 & - & 0,80 & - & 0,70 & 0,82 \\
\hline & $D$ & 0,33 & 0,41 & 0,34 & 0,49 & 0,44 & 0,33 \\
\hline \multirow[t]{2}{*}{ Collection } & $H^{\prime}$ & 0,60 & 0,65 & $\mathbf{0 , 8 0}$ & 0,47 & 0,99 & 0,93 \\
\hline & $D$ & 0,61 & $\mathbf{0 , 5 0}$ & 0,36 & 0,64 & 0,24 & 0,28 \\
\hline
\end{tabular}

\footnotetext{
$H^{\prime}$ : Indice de diversité de Shannon Weaver normalisé ;

(-) : Valeur de $H^{\prime}$ non déterminée car apparition de fréquence nulle $\left(\frac{\mathrm{Ni}}{\mathrm{N}}=0\right)$ pour une modalité ;

$D$ : Indice de diversité de Simpson.
} 


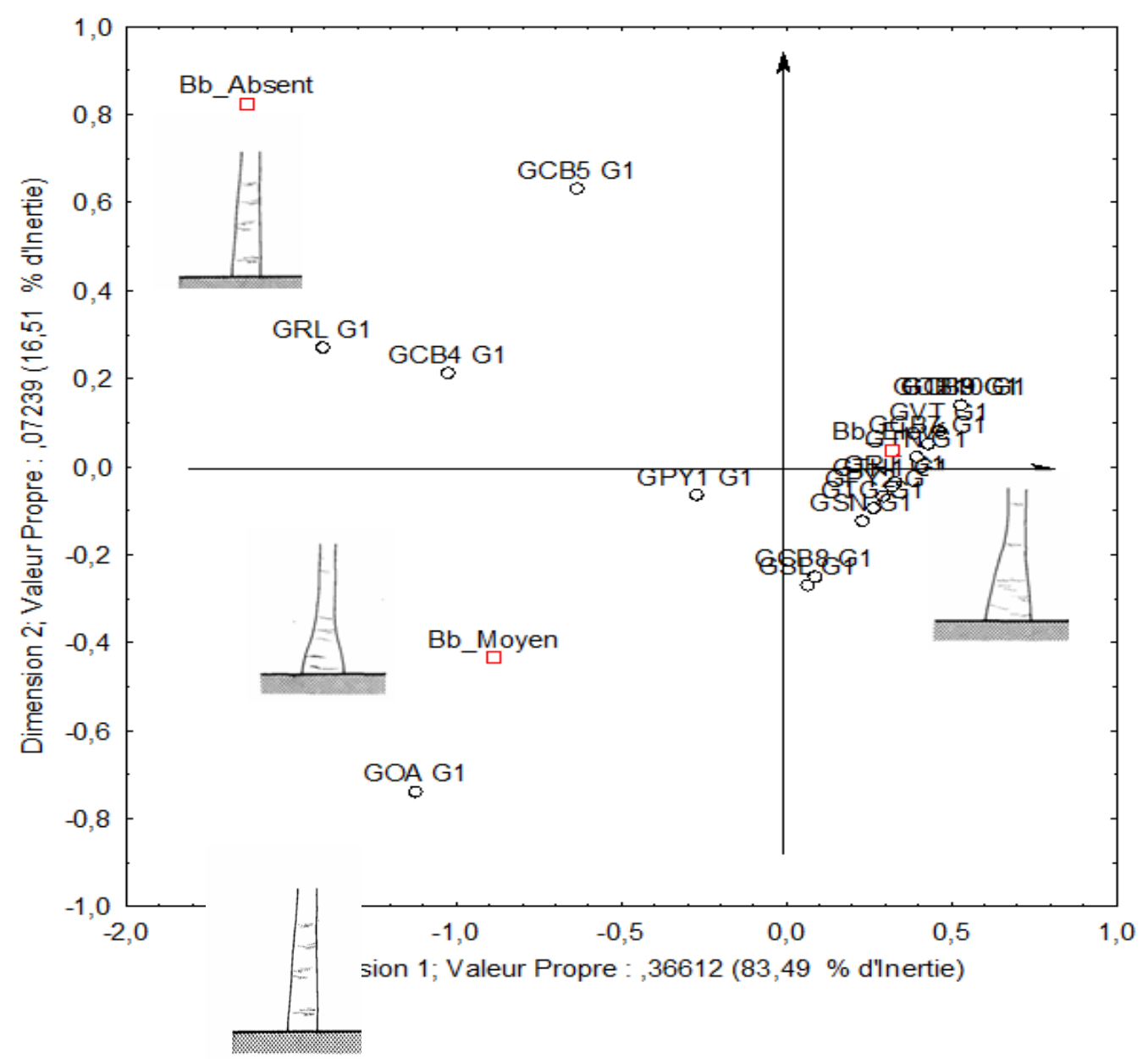

Figure 2 : Répartition de 18 accessions régénérées de cocotier Grand de la Collection Internationale pour l'Afrique et l'Océan Indien dans le plan formé par les dimensions 1 et 2 de l'AFC réalisée avec le caractère catégorie du bulbe.

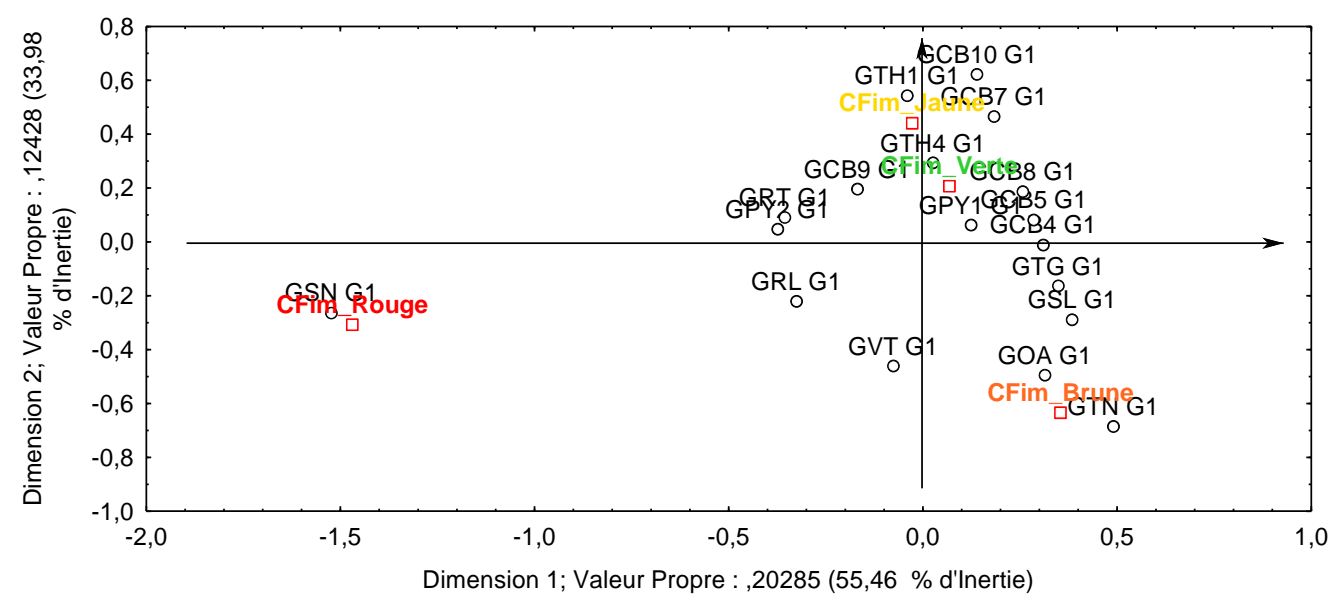

a. Couleur fruit immature. 


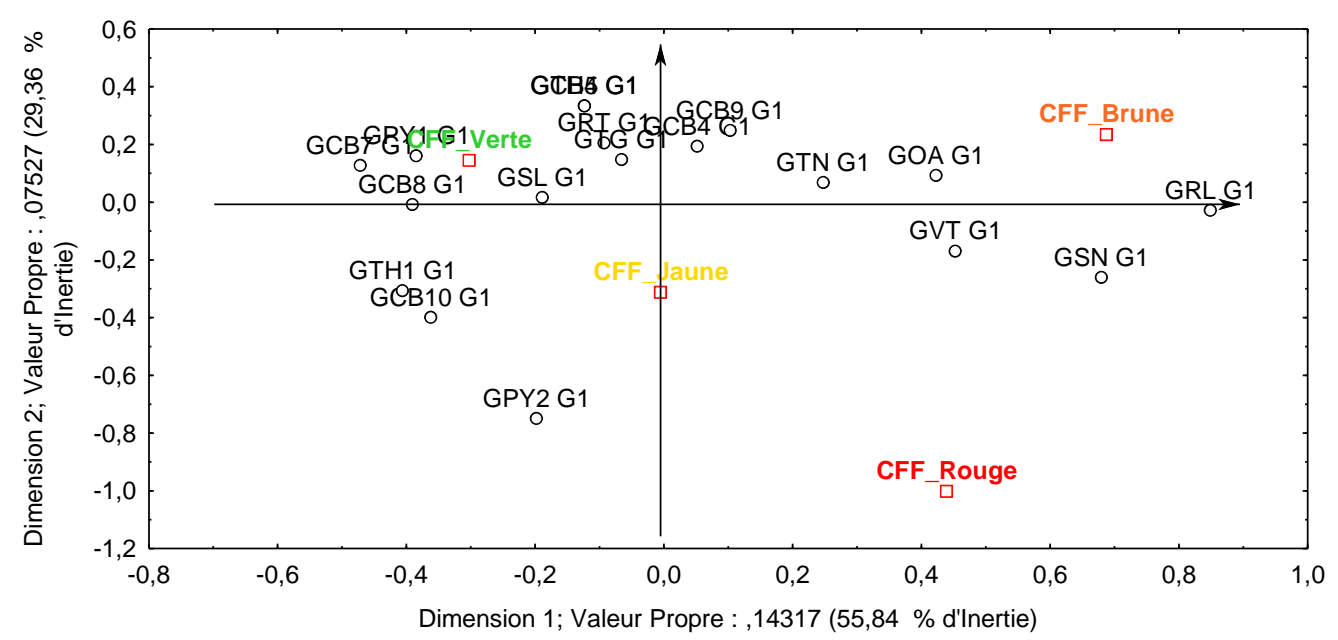

b. Couleur fleurs femelles.

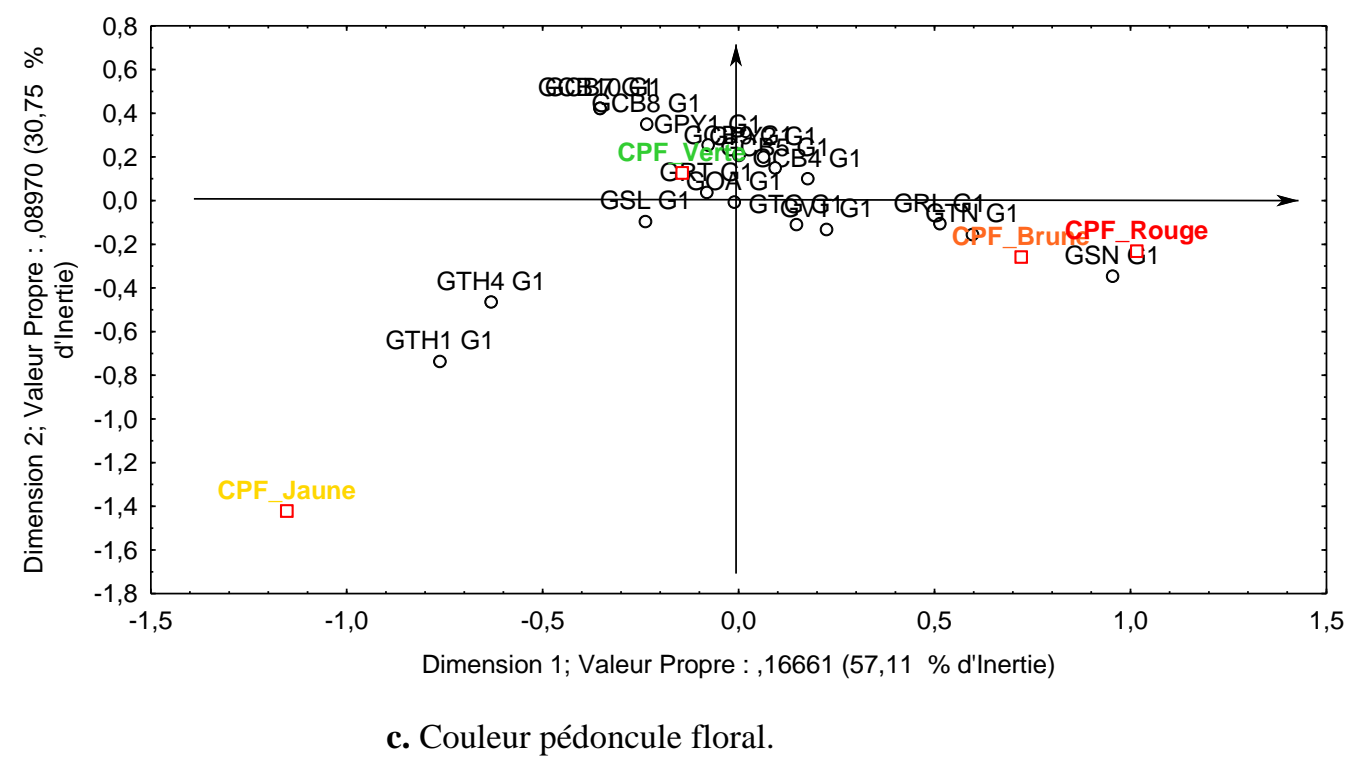

Figure 3 : Répartition de 18 accessions régénérées de cocotier Grand de la Collection Internationale pour l'Afrique et l'Océan Indien dans le plan formé par les dimensions 1 et 2 de l'AFC réalisée avec les caractères couleurs (a) du fruit immature, (b) des fleurs femelles et (c) du pédoncule floral. 


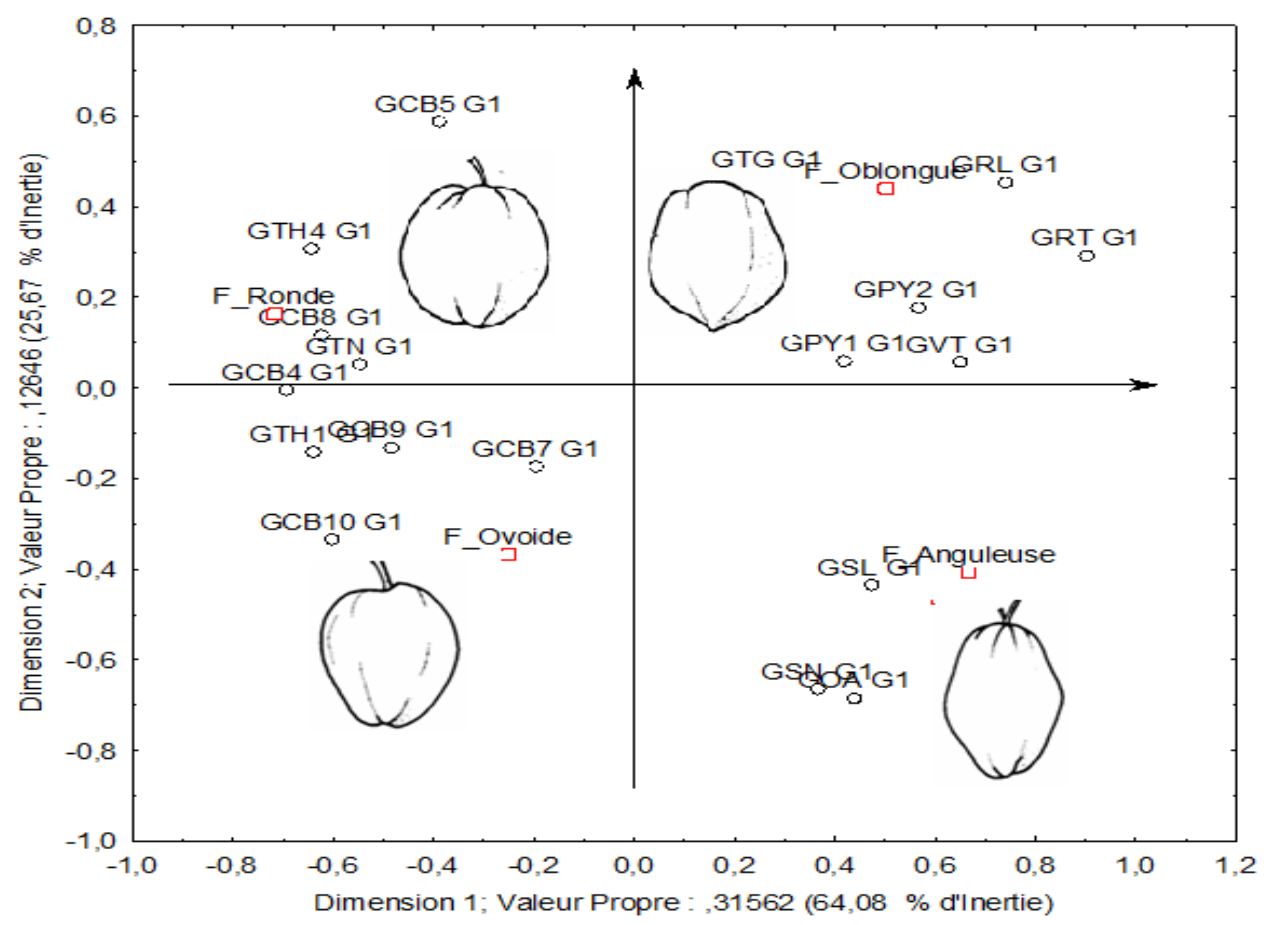

Figure 4 : Répartition de 18 accessions régénérées de cocotier Grand de la Collection Internationale pour l'Afrique et l'Océan Indien dans le plan formé par les dimensions 1 et 2 de l'AFC réalisée avec le caractère forme du bulbe.

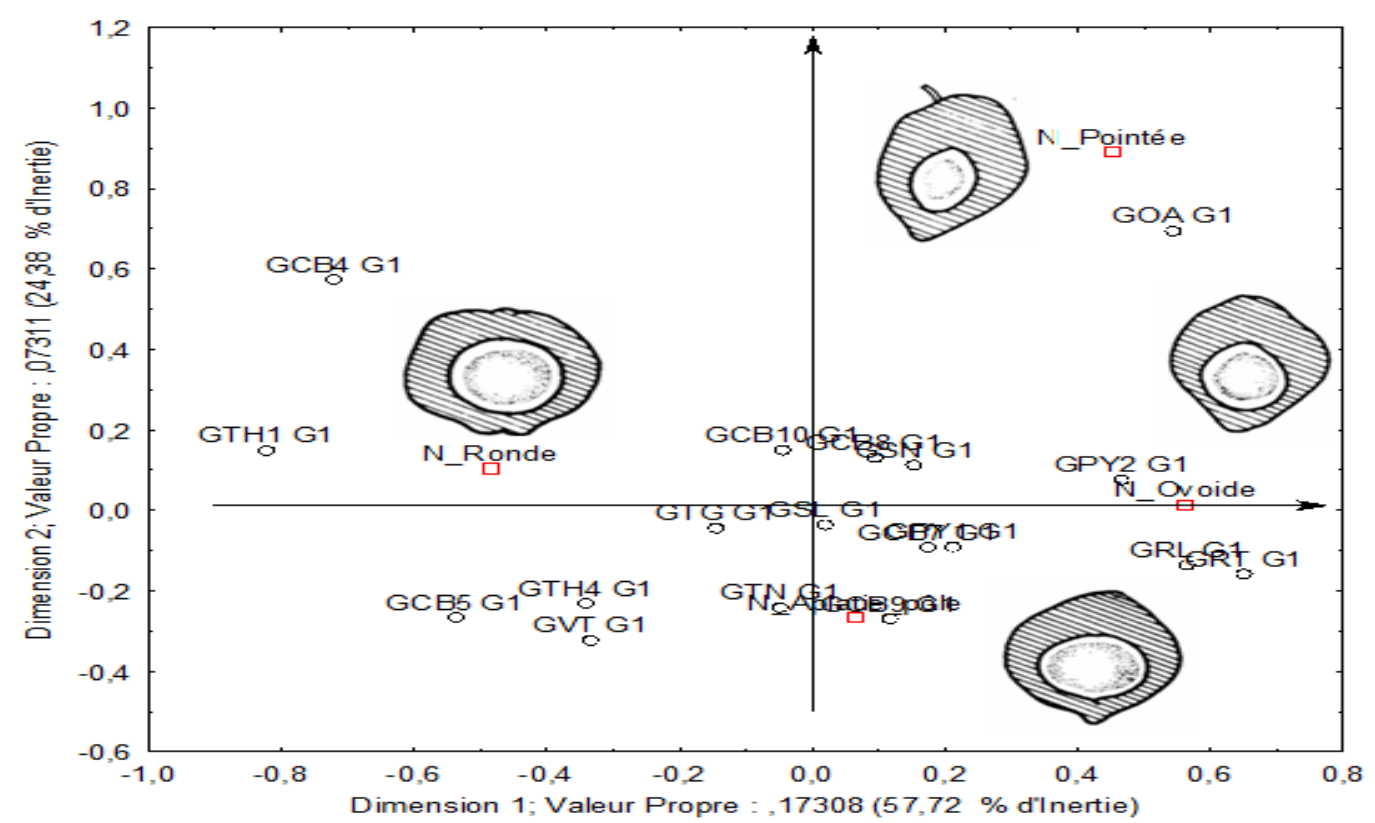

Figure 5 : Répartition de 18 accessions régénérées de cocotier Grand de la Collection Internationale pour l'Afrique et l'Océan Indien dans le plan formé par les dimensions 1 et 2 de l'AFC réalisée avec le caractère forme de la noix débourrée. 


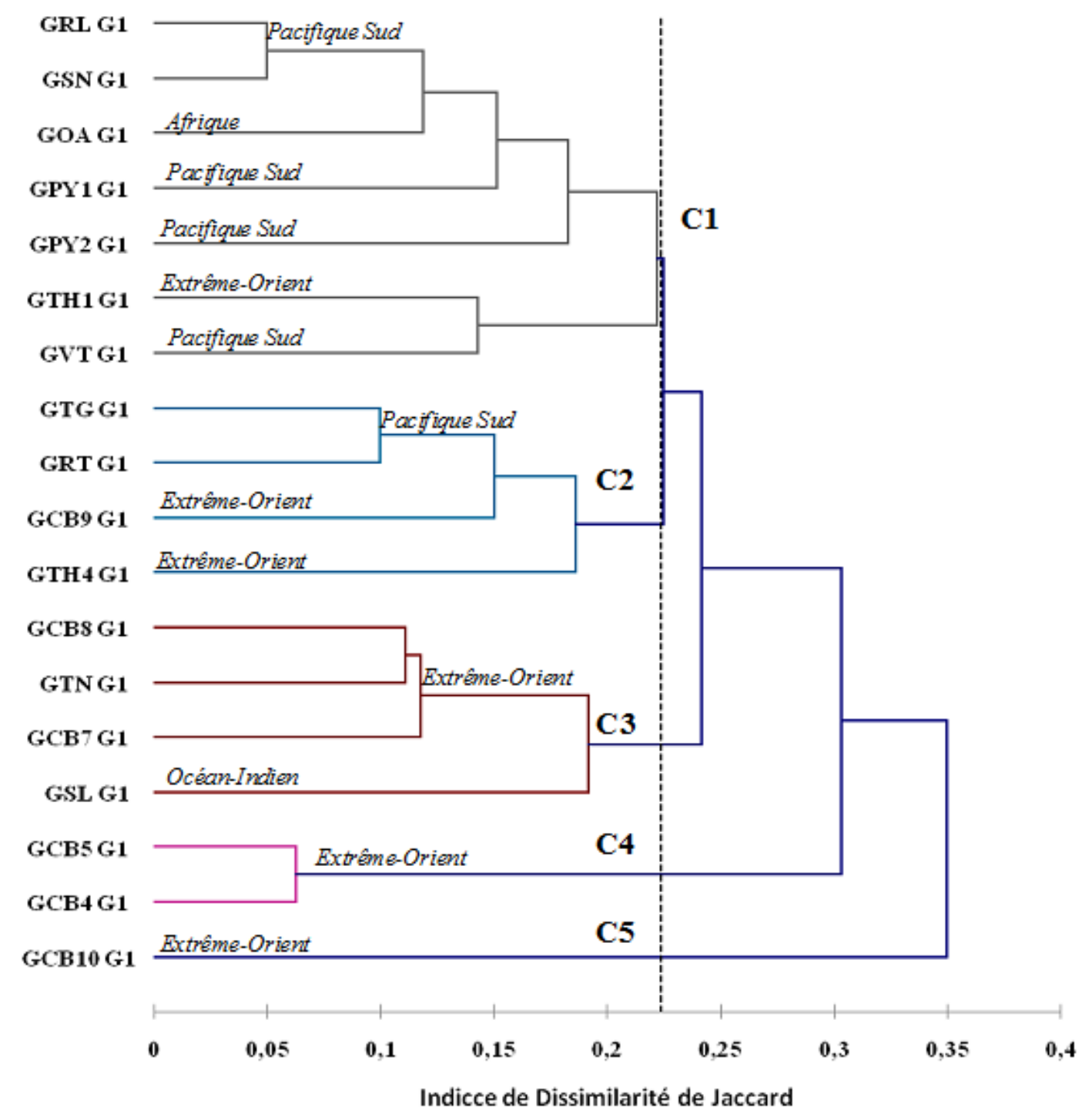

Figure 6: Dendrogramme construit à partir de l'indice de Jaccard révélant la structure de 18 accessions régénérées de cocotier Grand de la Collection Internationale pour l'Afrique et l'Océan Indien suivant l'expression de six caractères qualitatifs observés sur le stipe, l'inflorescence et le fruit.

\section{DISCUSSION}

Les résultats ont montré que les six caractères qualitatifs mesurés sur le stipe, l'inflorescence et le fruit ont présenté une variabilité intra et inter population plus ou moins importante. Cela témoigne que chez les cocotiers Grand le polymorphisme des caractères qualitatifs est fonction de la population surtout que d'après Konan et al. (2007a) le niveau d'hétérozygotie est variable chez ces populations qui sont allogames. Egalement, sur le karité (Diarrassouba et al., 2009), le figuier (Aljane et Ferchichi, 2014) et le fonio (Saidou et al., 2014) les caractères qualitatifs ont été utilisés pour révéler la variabilité phénotypique. En se basant sur des caractères qualitatifs tels que la catégorie du bulbe, la forme du fruit et la couleur du fruit immature, des populations de cocotier Grand ont été collectées dans les îles Andaman et 
Nicobar par le National Bureau of Plant Genetic Resources (NBPGR) pour être conservées en Inde (Abraham et al., 2008). Mais, en considérant à la fois les valeurs d'indices de diversité de Shannon Weaver normalisé $\left(H^{\prime}\right)$ et de Simpson $(D)$, respectivement proches de 1 et 0 , les deux caractères que sont les formes du fruit et de la noix débourrées ont été les plus discriminants au niveau intra et inter-population comme rapportés par Yao et al. (2015). De façon pratique, la forme de la noix mature, facilement observable serait plus appropriée pour l'identification des populations de cocotier Grand que la forme de la noix débourrée. Ce dernier caractère n'est observable qu'après le débourrage de la noix entière. Les descripteurs relatifs à la catégorie du bulbe, aux couleurs du fruit immature, des fleurs femelles et du pédoncule floral, compte tenu de leurs faibles valeurs de $H^{\prime}$ ' et fortes valeurs de $D$, peuvent être considérés comme secondaires. Concernant, la coloration de l'inflorescence, il faut prendre soin de bien définir le rang de l'inflorescence à observer sur l'arbre. En effet, chez le cocotier, l'inflorescence nouvellement épanouie de rang 10 a ses pièces florales majoritairement de couleur jaune et des biais d'observations ne sont pas à exclure si ce rang est observé.

Au niveau de la gestion d'une collection ex-situ de cocotier planté au champ, les résultats ont permis de ressortir certains phénotypes propres aux populations de cocotier Grand permettant leur identification. Le dendrogramme a montré que la typologie des populations de cocotier Grand générée à partir des caractères qualitatifs est en désaccord avec la structuration admise et fournie antérieurement à l'aide des marqueurs quantitatifs et moléculaires de type Simple Sequence Repeat (SSR). En effet, la typologie des accessions de cocotier Grand au sein de la collection de Côte d'Ivoire à l'aide de caractères quantitatifs (Konan 2008; Yao, 2014) et de marqueurs SSRs (Konan, 2008) a révélé deux principaux groupes. Il s'agit d'une part des accessions originaires d'Afrique et de l'Océan indien et d'autre part des accessions natives du Pacifique Sud, de l'Extrême Orient et d'Amérique latine. Le dendrogramme a aussi montré que certaines populations de cocotier Grand collectées dans le même pays comme les populations Grand de Cambodge (GCB) et Grand de Thaïlande (GTH) n'ont pas été classifiées dans le même groupe. La classe 1 composée des populations GOA G1, GRL G1, GSN G1, GPY1 G1, GPY2 G1, GTH1 G1 et GVT G1 présente les individus les plus variables dans l'expression des modalités des six caractères qualitatifs étudiés. En conséquence, il est difficile de différencier ces populations les unes des autres à l'aide des caractères étudiés. La classe 2 comprenant les populations GTG G1, GRT G1, GCB9 G1 et GTH4 G1 présente les individus ayant seulement des bulbes moyen et élevé. Le phénotype rouge de la coloration des fleurs femelles et du pédoncule floral n'a pas été identifié chez ces individus. Comme ceux de la classe 2, chez les individus de la classe 3 se comptant au sein des populations GCB7 G1, GCB8 G1, GTN G1 et GSL G1 la catégorie bulbe absent n'a pas été identifiée. La spécificité des individus de la classe 3 est la couleur des fruits immatures et du pédoncule floral qui varie entre le vert plus répandu et le brun. La classe 4 a été formée des deux populations du Cambodge GCB4 G1 et GCB5 G1 les plus variables suivant la catégorie du bulbe comme les populations de la classe 1. Les individus de la classe 4 n'ont pas donné des fruits anguleux et des noix débourrées ovoïdes. Ils ont présenté les phénotypes vert plus répandu et brun du fruit immature et du pédoncule floral. La coloration rouge des fleurs femelles est absente chez ces individus comme ceux de la classe 2. La population du Cambodge GCB10 G1 qui a constitué à elle seule la classe 5 a été homogène dans l'expression de la catégorie du bulbe (bulbe élevé) et de la coloration du pédoncule florale (couleur verte). Les fleurs femelles et le pédoncule floral n'ont présenté que les deux phénotypes vert et jaune.

Des critères d'identification faciles comme la forme du fruit et la couleur du fruit immature mis en évidence dans certaines populations de cocotier Grand peuvent assister le gestionnaire d'une collection au champ 
pendant sa régénération pour déceler des individus illégitimes comme rapportés par De Nuce et Wuidart. (1979). De même, ces critères peuvent être utilisés pour suivre l'identité des accessions de cocotier Grand pendant leur régénération. Concernant la forme du fruit, les populations de premier cycle de régénération (G1) de cocotier Grand de Cambodge (GCB4 G1, GCB5 G1 et GCB8 G1), GTH G1, GRL G1, GTG G1 et GRT G1 sont semblables aux populations initialement introduites (G0) dans la collection de Côte d'Ivoire. En effet, la dominance de la forme ronde du fruit mature a été rapportée chez les accessions parentales GCB G0 et GTH G0 (De Nuce et Wuidart, 1981 ; Sangaré et al., 1984). Dans ces travaux antérieurs, la forme oblongue du fruit mature citée chez les populations parentales GRL G0, GTG G0 et GRT G0 a été plus répandue (De Nuce et Wuidart, 1981 ; Sangaré et al., 1984). Cependant, la forme oblongue du fruit qui a plutôt caractérisé les populations parentales GSN G0 (De Nuce et Wuidart, 1981) et GOA G0 (De Nuce et al., 1970) a varié entre les formes anguleuse et ovoïde dans la génération régénérée. Cette différence s'expliquerait par le fait que sur les accessions parentales, la forme oblongue (plus longue que large) du fruit a été déduite du rapport entre les diamètres polaire et équatorial. Ces deux derniers caractères sont de types quantitatifs et non qualitatifs.

A propos de la couleur du fruit immature, les populations régénérées GTH G1 et GCB G1 où la couleur verte est dominante sont conforment à leurs homologues parentales antérieurement étudiées (De Nuce et Wuidart, 1981 ; Sangaré et al., 1984). Cependant, chez les populations régénérées GRL G1, GSN G1, GTG G1 et GRT G1, des colorations du fruit immature variant entre le vert, le brun et le rouge sont présentes alors que chez les populations parentales dont elles dérivent la dominance de la coloration brune de la noix immature est seulement citée (Nuce et Wuidart, 1981; Sangaré et al., 1984). Cette nonconformité aux populations initiales (G0) s'expliquerait par le fait que la sélection aléatoire de certains individus comme géniteurs au sein des populations de cocotier
Grand pendant leur régénération par voie sexuée aurait provoqué la réduction ou l'augmentation des fréquences des modalités dans les accessions régénérées. Cela peut aboutir soit à la disparition soit à la fixation d'un phénotype donné. Des variations semblables de fréquences alléliques entre les accessions initiales (G0) et régénérées (G1) de cocotier Grand ont été rapportées par Yao et al. (2013) à l'aide de marqueurs moléculaire de type SSR.

Au niveau de l'amélioration végétale, il est prouvé à l'aide des caractères quantitatifs que les populations de cocotier produisant des fruits allongés donnent un nombre élevé de fruits par régime car ceux-ci y occupent moins d'espace (Bourdeix et al., 2005). Il semblerait donc que les deux gènes contrôlant l'expression des caractères « taille du fruit » et «nombre de noix par régime» se trouvent sur le même groupe de liaison chez le cocotier. La forme du fruit étant très variable au sein des populations de cocotier Grand, il serait donc judicieux pour le sélectionneur de choisir les individus donnant spécifiquement des fruits de formes anguleuse et oblongue comme géniteurs pour optimiser les chances d'introgression de gènes de rendement élevé en fruits chez les lignées et hybrides améliorés. De même, la variabilité des couleurs de fruit immature observée chez les accessions de cocotier Grand serait source de diversité génétique. En effet, Koffi et al. (2013) ont suggéré que la diversité des couleurs de fruit observée parmi les individus hybrides F1 Nain Jaune Malaisie x Grand Vanuatu pourrait provenir d'une expression diverse de gènes, eux-mêmes à l'origine d'une tolérance variable à la maladie du Jaunissement Mortel (JM) du cocotier. De telles conclusions ont été tirées par Konan et al. (2007b) qui ont montré que les individus Grand Vanuatu sont diversifiés au plan moléculaire à l'aide de marqueurs microsatellites. Chez le cocotier, la variabilité élevée des couleurs du fruit et de l'inflorescence au sein d'une même population pourrait être à l'origine de la résistance de nombreux écotypes de cocotier Grand aux ravageurs et maladies. Konan et al. (2006) ont rapporté que les écotypes Grand de cocotier, 
contrairement aux écotypes Nain, renferment des gènes qui leurs confèrent une résistance aux maladies, ravageurs et stress hydrique compte tenu de leur niveau élevé d'hétérozygotie.

\section{Conclusion}

L'objectif de la présente étude a été de décrire et de révéler la typologie, à l'aide de six caractères morphologiques de type qualitatif de 18 populations de cocotier Grand plantées dans la collection internationale de Côte d'Ivoire. Une variabilité plus ou moins importante a été détectée tant au sein qu'entre les 18 populations de cocotier Grand. Les caractères formes du fruit et de la noix débourrée ont été les plus discriminants. Le dendrogramme a montré que la typologie des populations de cocotier Grand générée à partir de l'ensemble des caractères qualitatifs est en désaccord avec la structuration admise et fournie antérieurement à l'aide des marqueurs quantitatifs et SSRs. Ainsi, l'emploie des caractères morphologiques de type qualitatif, bien que longtemps marginalisée dans l'étude de la diversité génétique des populations de cocotier Grand, a donné des résultats pertinents à prendre en compte à la fois dans les programmes d'amélioration et de régénération des collections au champ.

\section{CONFLIT D'INTERETS}

Les auteurs déclarent qu'ils n'ont pas de conflit d'intérêts par rapport à cet article.

\section{CONTRIBUTIONS DES AUTEURS}

SDMY a rédigé le manuscrit ; EBZK a réalisé les traitements statistiques; MPWD et KY ont participé à la collecte des données ; JLKK a initié les travaux ; ND, RB et RSS ont supervisé les travaux et fait la relecture du manuscrit.

\section{REMERCIEMENTS}

Les données de cet article ont bénéficié pour leur collecte en 2015 de l'appui financier et technique de la Division Amélioration génétique du cocotier de la station Marc Delorme de Côte d'Ivoire qui héberge la collection internationale de cocotier pour l'Afrique et l'Océan Indien.

\section{REFERENCES}

Abraham Z, Senthikumar R, John K, Sharma S, Nair V, Unnikrishnan $\mathrm{P}$, Kumaran $\mathrm{P}$, George K, Uma S, Latha M, Malik S, Mishra K, Bhandari C, Pareek S. 2008. Collection of plant genetic resources from Andaman and Nicobar Islands. Genetic Resources and Crop Evolution, 55: 12791289. DOI : 10.1007/s10722-008-93279.

Aljane F, Ferchichi A. 2014. Sélection des accessions locales de figuier (Ficus carica L.) les plus performantes dans les régions arides tunisiennes. Revue des Régions Arides, 35 (3) : 39-46.

Arunachalam V, Jerad B, Damodaran V, Ratnambal M, Kumaran P. 2005. Phenotypic diversity of foliar traits in coconut gerplasm. Genetic Resources and Crop Evolution, 52: 1031-1037. DOI : 10.1007/s10722-004-6106-0.

Baudouin L, Santos G. 2005. Morphometric methods of determining diversity in coconut. In Coconut Genetic Ressources, Batugal P, Ramanatha V, Olivier J (Eds). IPGRI-APO:

Serdang, Malaysia; 209-223.

Bioversity international. 2008. Minimum list of descriptors for coconut. http: // WWW. Bioversityinternational.Org

Bourdeix R. 1988. Etude du déterminisme génétique de la couleur du germe chez le cocotier Nain. Oléagineux, 43(10) : 371374.

Bourdeix R, Konan JL, N'cho YP. 2005. Cocotier, Guide des Variétés Traditionnelles et Améliorées. Coproduction CIRAD /CNRA, édition diversiflora : Montpellier (France) ; 58 p.

Daramcoum WAMP, Yao SDM, Konan KJL, Koffi EBZ, Yoboue K, N'guetta ASP. 2017. Diversité agro-morphologique précoce d'hybrides d'autofécondation F3 de cocotier (Cocos nucifera L.) impliquant l'écotype Nain Vert Sri Lanka (NVS) tolérant à la maladie du Jaunissement Mortel. Journal of Applied 
Biosciences, 112: 11045-1105. DOI: https://dx.doi.org/10.4314/jab.v112i1.10.

De Nuce L, Wuidart W. 1979. Les cocotiers grands de Port Bouët (Côte d'Ivoire). 1Grand Ouest Africain, Grand de Mozambique, Grand de Polynésie, Grand de Malaisie. Oléagineux, 34(7): 339-349.

De Nuce L, Rognon F. 1977. Les cocotiers nains à Port-Bouët (Côte d'Ivoire). INain Jaune Ghana, Nain Rouge Malais, Nain Vert Guinée Equatoriale, Nain Rouge Cameroun.

Oléagineux, 32 (8-9) :367-375.

De Nuce L, Wuidart W, Sangaré, A. 1980. La fécondation artificielle du cocotier. Oléagineux, 35 (4) : 319-326.

De Nuce L, Wuidart W. 1981. Les cocotiers Grands à Port-Bouët (Côte d'Ivoire). 2Grand Rennell, Grand Salomon, Grand Thaillande, Grand Nouvelles-Hébrides. Oléagineux, 36 (7) : 353-362.

Diarrassouba N, Fofana I, Issali A, Bup N, Sangaré A. 2009. Typoloy of shea trees (Vitellaria paradoxa) using qualitative morphological trait in Côte d'Ivoire. Plant Genetic Resources Newsletter, 205: 10 15.

Ghesquière M. 1984. Polymorphisme enzymatique chez le palmier à huile (Elaeis guineensis Jacq.). I. Contrôle génétique de neuf systèmes enzymatiques. Oléagineux, 39 (12) : $\quad 561-574$.

Jay M, Bourdeix R, Potier F, Sanlaville C. 1989. Premiers résultats de l'étude des polyphénols foliaires du cocotier. Oléagineux, 44: 151-161.

Koffi EBZ, Konan JL, Issali A, Lekadou TT, Bourdeix R, Allou K, Zoro BI A. 2013. Evaluation de la diversité agromorphologique des descendances hybrides de cocotiers (Cocos nucifera $\mathrm{L}$.) Nain Jaune Malaisie x Grand Vanuatu en Côte d'Ivoire. International Journal of Biology and Chemical Sciences, 7(2): 507-522. DOI: http://dx.doi.org/10.4314/ijbcs.v7i2.8.

Koffi EBZ, Yao SDM, Sie RS, Konan KJL, Koffi Y, Doh F, Issali AE, Allou K. 2016. Morphological Diversity among 18
Genitors of Vanuatu Tall (VTT) Coconut (Cocos nucifera L.) Population used in Crosses for Hybrids Resistant to Lethal Yellowing Disease Selection at PortBouët, Côte d'Ivoire. Greener Journal of Agricultural Sciences, 6(4): 134-144. DOI:

http://doi.org/10.15580/GJAS.2016.4.02 0416032.

Koffi EBZ, Konan K JL, Yao SDM, Sié RS, Diarrassouba N. 2019. Study of the crossing of improved tall coconut $\mathrm{x}$ improved tall coconut in Côte d'Ivoire. Global Journal of Plant Breeding and Genetics, 6 (3): 484-493.

Konan JL, Bourdeix R, Sangaré A, Mondeil F. 2006. Caractérisation de quelques cultivars de cocotier (Cocos nucifera L.) tolérant à la sécheresse en Côte d'Ivoire. Agronomie Africaine, 18 (2) : 145-156. DOI : http://dx.doi.org/10.4314/aga.v18i2.168.

Konan JL, Bourdeix R, George ML. 2008. Directives pour la régénération : cocotier. In Crop Specific Regeneration Guidelines [CD-ROM], Dulloo ME, Thormann I, Jorge MA, Hanson J (eds). CGIAR System-wide Genetic Resource Programme (SGRP): Rome, Italy; 11 p.

Konan K, Konan K, Koffi K, Lebrun P, Sangaré A. 2007a. Coconut Microsatellite gene diversity analysis technology transfer to Côte d'Ivoire. Biotechnology, 3: 383-388. DOI: 10.3923/biotech.2007.383.388.

Konan K, Koffi K, Konan K, Lebrun P, Dery S, Sangaré A. 2007 b. Microsatellite gene diversity in coconut (Cocos nucifera L.) accessions resistants to lethal yellowing disease. African Journal of Biotechnology, 6(4): 341-347. DOI: 10.4314/ajb.v6i4.56209

Konan K. 2008. Evaluation de la diversité agromorphologique et moléculaire de la collection internationale de cocotier (Cocos nucifera L.) en Côte-d'Ivoire. PhD thesis, University of Cocody, Côte d'Ivoire, $150 \mathrm{pp}$.

Lebrun P, N'cho Y, Seguin M, Grivet L, Baudouin L. 1998. Genetic diversity in 
coconut (Cocos nucifera L.) revealed by restriction fragment length polymorphism (RFLP) markers. Euphytica, 101: 103108. DOI : https://doi.org/10.1023/A:101832372180 3.

Le Saint JP, De Nuce L, Sangaré A. 1983. Les cocotiers nains à Port-Bouët (Côte d'Ivoire). II- Nain Vert Sri Lanka et complément d'information sur les Nains Jaune et Rouge Malaisie, Vert Guinée Equatoriale et Rouge Cameroun. Oléagineux, 38 (11) : 594-601.

N'Cho YP, Le Saint JP, Sangaré A. 1988. Les cocotiers Nains à Port-Bouët (Côte d'Ivoire) III.- Nain Brun NouvelleGuinée, Nain Vert Thaïlande et Nain Rouge Polynésie. Oléagineux, $\mathbf{4 3}$ (2) : 55-64.

Sangaré A, Le Saint JP, De Nuce L. 1984. Les cocotiers Grands à Port-Bouët (Côte d'Ivoire). 3- Grand Cambodge, Grand Tonga, Grand Rotuma, Grand NouvellesHébrides. Oléagineux 39 (4) : 205-215.

Saidou S, Bakasso Y, Inoussa M, Zaman-Allah M, Atta S, Barnaud A, Billot C, Saadou M. 2014. Diversité agro-morphologique des accessions de fonio [Digitaria exilis (Kippist.) Stapf.] au Niger. International Journal of Biology and Chemical Sciences, 8 (4): 1710-1729. DOI: http://dx.doi.org/10.4314/ijbcs.v8i4.31.

StatSoft France. 2005. Statistica, logiciel d'analyse de données version 7.1. www.statsoft.fr.

Teulat B, Aldam C, Trehim P, Lebrun P, Barker J, Arnold G, Karp A, Baudouin L, Rognon F. 2000. An analysis of genetic diversity in coconut (Cocos nucifera $\mathrm{L}$.) population from across the geographical range using sequence tagged microsatellites (SSRs) and (AFLPs). Theoretical Applied Genetic, 100: 764 771.

DOI: https://doi.org/10.1007/s00122005135. Yao SDM, Konan JL, Pokou N, Konan K, Issali
A, Sié R, Zoro Bi A. 2013. Assessment of the genetic diversity conservation in three tall coconut (Cocos nucifera L.) accessions regenerated by controlled pollination, using microsatellite markers. African Journal of Biotechnology, 12 (20): $\quad 2808-2815 . \quad$ DOI: 10.5897/AJB11.3608.

Yao SDM. 2014. Impact de la régénération de la collection internationale de cocotier (Cocos nucifera L.) pour l'Afrique et l'Océan indien sur la diversité agromorphologique et moléculaire. $\mathrm{PhD}$ Thesis, University of Nangui Abrogoua, Côte d'Ivoire ; 162 pages.

Yao SDM, Konan JL, Sié R, Diarrassouba N, Lekadou TT, Koffi EBZ, Yoboue K, Bourdeix R, Issali A E, Doh F, Allou K, Zoro Bi IA. 2015. Fiabilité d'une liste minimale de descripteurs agromorphologiques recommandée par le COGENT dans l'étude de la diversité génétique du cocotier (Cocos nucifera L.). Journal of Animal and Plant Sciences, 26 (1): 4006-4022.

Yao SDM, Diarrassouba N, Dago DN, Koffi EBZ, Fofana IJ, Konan KJN, Konan KJL, Bourdeix R, Sie RS, Zoro BIA. 2019a. Genetic relationships among regenerated tall coconut (Cocos nucifera L.) accessions from multivariate analyses using reliable short list of morphological descriptors in Côte d'Ivoire. Journal of Plant Breeding and Crop Science, 11 (5): 137-143.

DOI: 10.5897/JPBCS2018.0788.

Yao SDM, Koffi EBZ, Konan NY, Konan KJL, Diarrassouba N, Fofana IJ, Konan KJN, Silué S, Sie RS, Bourdeix R. 2019b. Typology in the fertility of seven tall coconut (cocos nucifera 1.) populations using artificial pollination traits in field genebank. Journal of Plant Breeding and Genetics 7 (1): 33-40. DOI: 10.33687/pbg.007.01.2858. 